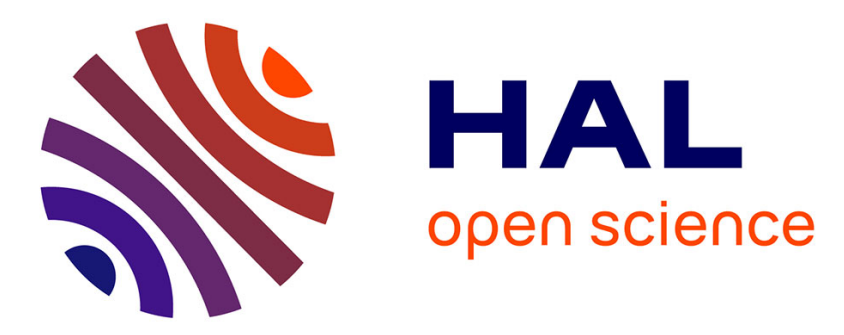

\title{
THEORETICAL STUDY OF HIGH INJECTION EFFECTS IN EBIC MEASUREMENTS OF GRAIN BOUNDARY RECOMBINATION VELOCITY IN SILICON
}

\author{
Jean-Luc Maurice, Y. Marfaing
}

\section{To cite this version:}

Jean-Luc Maurice, Y. Marfaing. THEORETICAL STUDY OF HIGH INJECTION EFFECTS IN EBIC MEASUREMENTS OF GRAIN BOUNDARY RECOMBINATION VELOCITY IN SILICON. Journal de Physique IV Proceedings, 1991, 01 (C6), pp.C6-77-C6-82. 10.1051/jp4:1991614 . jpa00250699

\section{HAL Id: jpa-00250699 https://hal.science/jpa-00250699}

Submitted on 1 Jan 1991

HAL is a multi-disciplinary open access archive for the deposit and dissemination of scientific research documents, whether they are published or not. The documents may come from teaching and research institutions in France or abroad, or from public or private research centers.
L'archive ouverte pluridisciplinaire HAL, est destinée au dépôt et à la diffusion de documents scientifiques de niveau recherche, publiés ou non, émanant des établissements d'enseignement et de recherche français ou étrangers, des laboratoires publics ou privés. 
JOURNAL DE PHYSIQUE IV

Colloque C6, supplément au Journal de Physique III, Vol. 1, décembre 1991

C $6-77$

\title{
THEORETICAL STUDY OF HIGH INJECTION EFFECTS IN EBIC MEASUREMENTS OF GRAIN BOUNDARY RECOMBINATION VELOCITY IN SILICON
}

\author{
J.-L. MAURICE and Y. MARFAING* \\ Laboratoire de Physique des Matériaux, CNRS-Bellevue, 1 Place Aristide-Briand, F-92195 \\ Meudon Cedex, France \\ *Laboratoire de Physique des Solides de Bellevue, CNRS-Bellevue, 1 Place Aristide-Briand, \\ F-92195 Meudon Cedex, France
}

\begin{abstract}
The presence of carriers in the grain boundary (GB) space charge is taken into account in solving numerically Poisson's equation. This allows one, by using a schematic description of electron excitation, to investigate the theoretical behaviour of the GB recombination velocity, measured by EBIC, in high injection conditions.
\end{abstract}

\section{Introduction.}

The electron beam induced current (EBIC) measurement of the recombination velocity $\left(v_{s}\right)$ at the grain boundaries (GB) in silicon is of interest for both fundamental research and photovoltaic industry. The link between $v_{s}$ and the physical parameters of the GB traps (density $N_{t}$, energy in the gap $E_{t}$ and capture cross sections for electrons and holes $\sigma_{n}$ and $\sigma_{p}$ ) is however quite complex. We recently proposed /1/ a set of relations allowing one to deduce these parameters from EBIC measurements of $v_{s}$ at varying temperature and injection level. However, for sake of simplicity, the presence of carriers in the GB space charge was either not considered (high barriers) or introduced very roughly (10w barriers) and the carrier transport in this zone was also described in a schematic way.

In this paper, the presence of charge carriers in the Space Charge Region (SCR) is fully taken into account in Poisson's equation and this, in turn, allows one to use rigorous expressions for the minority and majority carrier currents (the only neglected phenomena are injection and recombination in this region). While high injection is likely to frequently occur in EBIC experiments, the present treatment has the advantage to allow, for the first time, to investigate theoretically the effects of such excitation conditions on the GB recombination velocity. This is performed on two fictional grain boundaries representative of the ones existing in p-type large grain polysilicon. The previous simplified model is simultaneously applied and comparison with the present calculation indicates its validity limits.

2. Modelling of the presence of excess carriers in the grain boundary SCR.

Poisson's equation can be written

$$
\frac{d^{2} \varphi}{d x^{2}}=\frac{q^{2}}{\epsilon \epsilon_{0}}\left[p(x)-n(x)-N_{A}\right]
$$




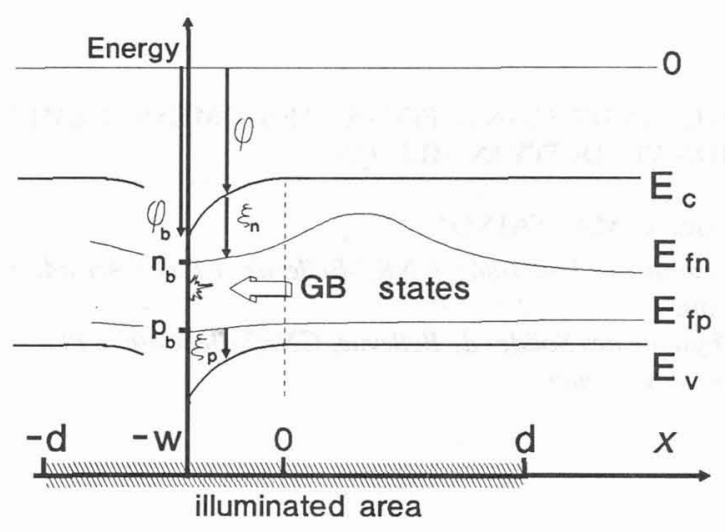

Fig.1. Energy level diagram in the vicinity of a grain boundary under local excitation. The $\mathrm{GB}$ is at $x=-W ; x=0$ corresponds to the edge of the $G B$ space charge region. Other notations are defined in the text (the curves are arbitrary and do not reflect a real situation).

where $\varphi$ is the electrostatic potential energy, $x$ the spatial coordinate (Fig.1), $q$ the elementary charge, $\epsilon \epsilon_{0}$ the silicon dielectric constant, and $N_{A}$ the acceptor concentration (we assume a p-type bulk material, form and results would be symmetrically identical for n-type). The existence of free carriers in the SCR is represented by the $x$-dependent concentrations of electrons $n(x)$ and holes $p(x)$,

$$
\begin{aligned}
& n(x)=N_{c} \exp \xi_{n} / k T, \\
& p(x)=N_{v} \exp \xi_{p} / k T,
\end{aligned}
$$

where $N_{c}, N_{v}$, are the densities of states at the conduction and valence band edges respectively, kT is Boltzmann's constant times the temperature and $\xi_{n}, \xi_{p}$ are the $x$-dependent chemical potentials of, respectively, electrons and holes.

Integrating equation (1) over electrostatic potential and using the Gauss theorem allows one to derive the charge of the zone (on one side of the GB) :

$$
\begin{aligned}
Q_{S C} & =-\frac{\epsilon \epsilon_{0}}{q}\left(\frac{d \varphi}{d x}\right) \varphi_{b} \\
& =\left[2 \epsilon \epsilon_{0}\left(N_{v} \int_{0}^{\varphi_{b}} \exp \frac{\xi_{p}}{k T} d \varphi-N_{c} \int_{0}^{\varphi_{b}} \exp \frac{\xi_{n}}{k T} d \varphi-N_{A} \varphi_{b}\right)\right]^{1 / 2}
\end{aligned}
$$

where $\varphi_{b}$ is the barrier energy height $\left(\varphi_{b}=-q \phi_{b i}\right.$ of ref./l/). The electron current in the SCR is

$$
i_{n}=\mu_{n} n \frac{d E_{F n}}{d x}
$$

where $E_{F n}$ is the electron quasi Fermi level, $E_{F n}=\varphi+\varepsilon_{n}$. This expression can be put in the form : 


$$
i_{n}=\mu_{n} N_{c} \exp \frac{\xi_{n}}{k T}\left(1+\frac{d \xi_{n}}{d \varphi}\right) \frac{d \varphi}{d x} .
$$

In steady state, this current is exactly compensated by the hole current which takes a similar form :

$$
i_{p}=\mu_{p} N_{v} \exp \frac{\xi_{p}}{k T}\left(1-\frac{d \xi_{p}}{d \varphi}\right) \frac{d \varphi}{d x} ;
$$

$\mu_{n}$ is the minority carrier electron mobility and $\mu_{\mathrm{p}}$ is the standard hole mobility. Equation (3) has to be integrated numerically by varying $\varphi$. One then needs to know $\xi_{n}(\varphi)$ and $\xi_{p}(\varphi)$. This is obtained by extracting $d \xi_{n, p} / d \varphi$ from eqs (5a and $\left.5 b\right)$ :

$$
\begin{aligned}
& \left(\frac{d \xi_{n}}{d \varphi}\right)_{\varphi}=\frac{i_{n}}{\left(\mu_{n} N_{c} \exp \frac{\xi_{n}}{k T}\right)(d \varphi / d x)}-1, \\
& \left(\frac{d \xi_{p}}{d \varphi}\right)_{\varphi}=\frac{i_{n}}{\left(\mu_{p} N_{v} \exp \frac{\xi_{p}}{k T}\right)(d \varphi / d x)}+1,
\end{aligned}
$$

where we have used the relation $i_{n}+i_{p}=0$. The quantity $\left(\frac{d \varphi}{d x}\right)_{\varphi}$ is deduced from eq. (3) taking $\varphi_{\mathrm{b}}=\varphi$.

\section{Current continuity and electrical neutrality.}

The current given in (5a) is equal by continuity on the one hand to the recombination current (i) at the GB which is related to the physical parameters $N_{t}$, $E_{f}, \quad \sigma_{n}, \sigma_{p}$, and on the other hand to the current (ii) coming from the grain. Using Shockley Read Hall (SRH) theory $/ 3,4$, current (i) is :

$$
i_{n}=\frac{N_{t}}{2} r_{p}\left(p_{b} n_{b}-n_{i}^{2}\right) /\left[n_{b}+n_{1}+a\left(p_{b}+p_{1}\right)\right]
$$

where $a=\gamma_{p} / \gamma_{n}, \gamma_{p}=v_{p} \sigma_{p}, \gamma_{n}=v_{n} \sigma_{n}$, with $v_{n}, v_{p}$, the thermal velocities of electrons and holes respectively; $p_{b}, n_{b}$ are the carrier concentrations at the GB and $n_{1}, p_{1}$ are respectively the electron and hole concentrations that would exist if the Fermi level were at the trap energy $E_{t}$. The current from the grain (ii) is solution of the diffusion equation for $x>0 / 1 /$ and makes the link with both $v_{s}$ and the excitation rate $\mathrm{g}$ :

$$
i_{n}=q v_{s} \Delta n_{0}=q \frac{D_{n}}{L_{n}}\left[g \frac{L^{2} n}{D_{n}}\left(1-\exp \frac{-d}{L_{n}}\right)-\Delta n_{0}\right] ;
$$

$\Delta n$ is the excess carrier concentration at the edge of the space charge, $D_{n}$ is the minority electron diffusivity, $L_{n}$ is the electron diffusion length and $d$ is the 
radius of the generation sphere $/ 2 /$,

$$
d(\mu \mathrm{m})=\frac{1}{2} \times 0.0171\left[E_{b}(k e V)\right]^{1.75},
$$

where $E_{b}$ is the beam energy.

The generation rate $g$ is taken constant inside the interval [-d, $+d]$ - except in the SCR where it is not taken into account - and null outside. The g value can be derived from the beam current $I_{b}$ and the beam voltage $E_{b}$ by assuming a uniform generation within the generation sphere :

$$
g=\frac{I_{b}}{q} \frac{E_{b}(k e V)}{3.7 \times 10^{-3}}\left(\frac{4}{3} \pi d^{3}\right)^{-1}
$$

where $3.7 \mathrm{eV}$ is the electron hole pair creation energy.

Quite generally, electrical neutrality implies that the total charge of the SCR, 2 $x \quad Q_{S G}$, is equal and opposite to the charge trapped at the GB which is given by the Schokley-Real-Hall expression /3,4/ (for a donor-like recombination centre) :

$$
Q_{G B}=q N_{t} \frac{n_{1}+a p_{b}}{a\left(p_{1}+p_{b}\right)+n_{1}+n_{b}} .
$$

\section{Solving the problem.}

The parameters are $E_{t}, N_{t}, \sigma_{n}, \sigma_{p}, T, E_{b}, I_{b}$ but only the first four are actual unknowns. However to get useful curves, we fix all of them except the beam current $I_{b}$ which is allowed to increase regularly over a given range. The algorithm for a given $I_{b}$ value proceeds as follows :

i) a trial value is given to $\Delta n_{0}, i_{n}$ is then calculated from (8).

ii) the starting values in the integration process are $\varphi=0, \xi_{\eta_{0}}\left(n_{0}+\Delta n_{0}\right), \varepsilon_{p}\left(p_{0}\right.$ $\left.+\Delta n_{0}\right)$. The initial derivatives of $\varphi, \xi_{n}$ are calculated using $(3),(6)$. Then $\varphi$ is given a small increment $(-d \varphi)$ and new values of $\xi_{n}, \xi_{p}$ are deduced from the above equations. The process is repeated.

iii) At each step $j, Q_{s c}$ (eq.3) is compared to the quantity $Q_{G B}$ (obtained by putting $n_{j}=n_{b}$ and $p_{j}=p_{b}$ in eq.11). If $2 \times Q_{s c}=Q_{G B}$, the integration over $\varphi$ is terminated and the computer proceeds to iv.

iv) A new value of $i_{\eta}$ is calculated using the expression of the recombination current at the GB ?eq.(7)), from which a new value of $\Delta n_{\text {g }}$ is deduced through (8). The whole process is iterated until the first and final values of $\Delta n_{0}$ are equal.

Finally, $v_{s}$ is calculated using (8).

\section{Practical results and conclusions.}

The two systems chosen are grain boundaries in $p$-type silicon $\left(p_{0}=10^{16} \mathrm{~cm}^{-3}, L_{n}=\right.$ $30 \mu \mathrm{m})$, with the following trap characteristics and operating conditions $E_{t}=E_{v}^{n}+$ $0.45 \mathrm{eV}, \sigma_{n}=\sigma_{p}=10^{-15} \mathrm{~cm}^{2}, T=300 \mathrm{~K}, E_{b}=20 \mathrm{keV}$. The trap density $N_{t}$ is put 


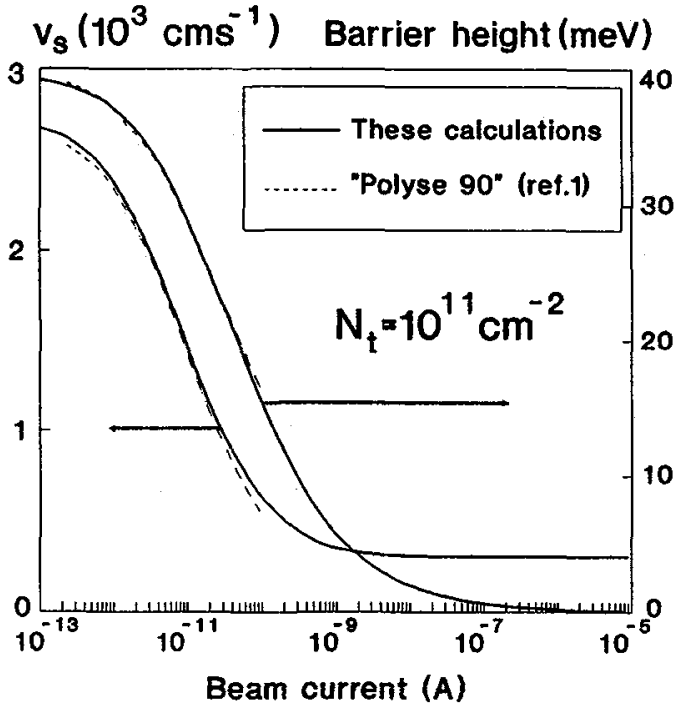

(a)

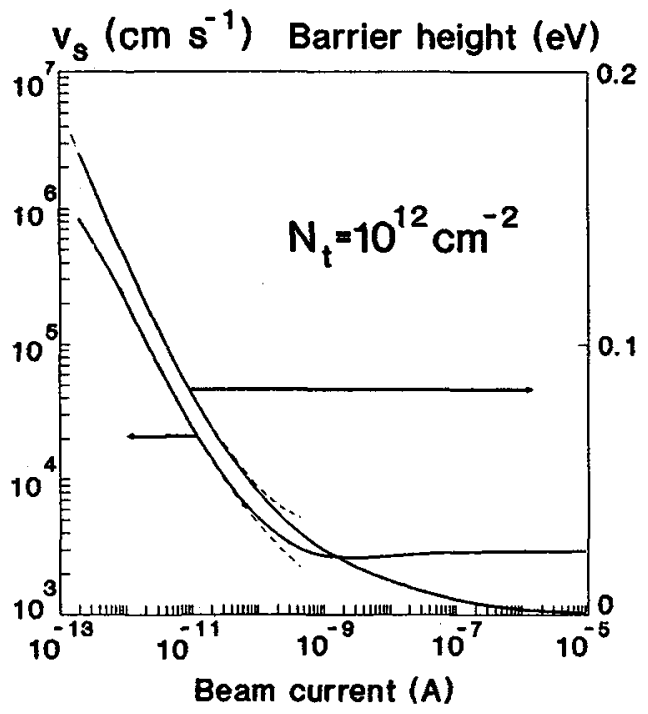

(b)

Fig.2. Recombination velocity $v_{s}$ and barrier height $\varphi_{q_{1}}$ as a function of beam current $I_{b}$ at a beam energy of $20 \mathrm{keV}$, for (a) a GB with a density of hole traps near mid-gap of $N_{t}=10^{11} \mathrm{~cm}^{-2}\left(\varphi_{b \circ}=0.039 \mathrm{eV}\right)$ and $(b)$ a $G B$ with $N_{t}=10^{12} \mathrm{~cm}^{-2}\left(\varphi_{b o}\right.$ $=0.28 \mathrm{eV}),(T=300 \mathrm{~K}, \mathrm{kT}=0.025 \mathrm{eV})$.

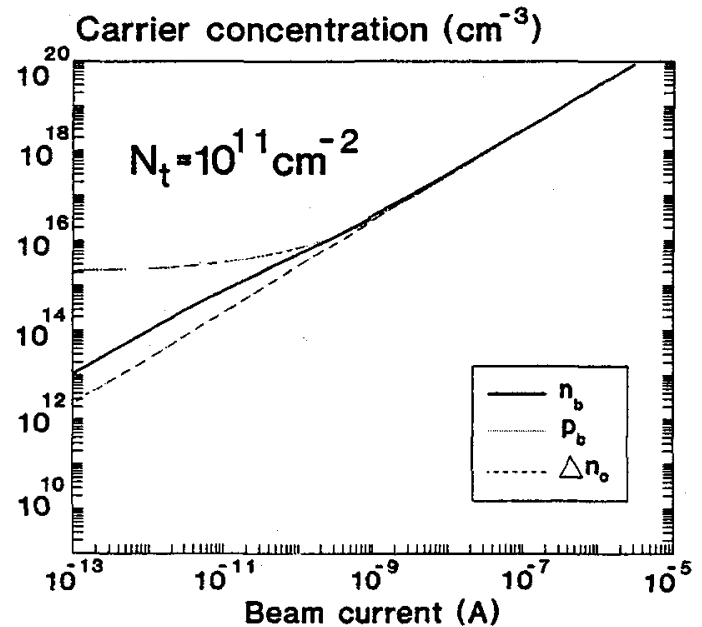

(a)

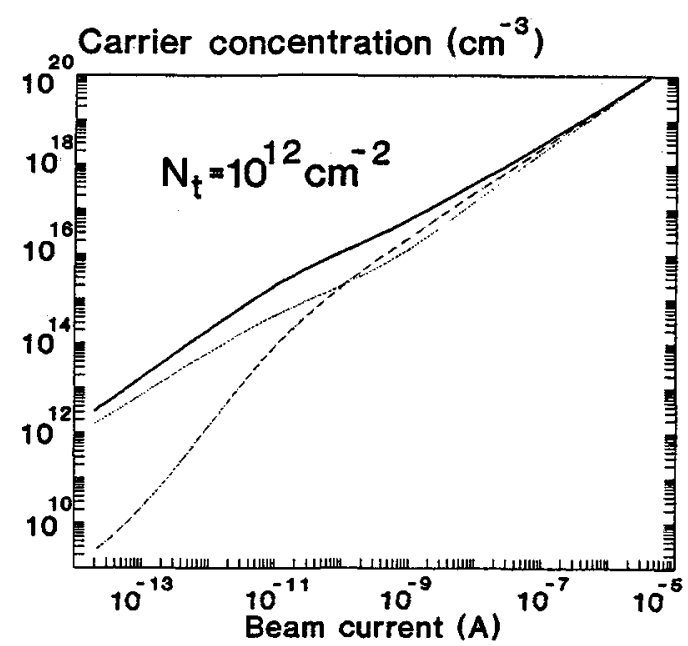

(b)

Fig.3. Concentrations of electrons $\left(n_{b}\right)$ and holes $\left(p_{b}\right)$ at the $G B$, and of excess carriers $\left(\Delta n_{0}\right)$ at the edge of the $G B$ space charge region, as a function of beam current $I_{k}$, for (a) the low barrier-GB $\left(\varphi_{b o}=0.039 \mathrm{eV}\right)$ and $(b)$ the high barrier-GB $\left(\varphi_{\mathrm{b}}=0.28 \mathrm{eV}\right)$. The equilibrium concentration of holes in the grains is $P_{0}=$ $10^{16} \mathrm{~cm}^{-3}$; in case (b) the equilibrium values at the $G B$ are $n_{b o}=10^{9} \mathrm{~cm}^{-3}$ and $p_{b_{0}}=$ $2 \times 10^{11} \mathrm{~cm}^{-3}$. 
equal to $10^{11} \mathrm{~cm}^{-2}$ (example (a)) and to $10^{12} \mathrm{~cm}^{-2}$ (example (b)) which correspond respectively to equilibrium barrier heights of $0.039 \mathrm{eV}$ and $0.285 \mathrm{eV}$ considering the trap states as single donors. These values have been chosen to match those generally found in the litterature /5/. The calculations have been performed over a range of beam current wider than that of standard electron microscopes by more than one order of magnitude on the low as well as on the high range sides. The behaviour of recombination velocity $v_{s}$ and barrier height $\varphi_{b}$ as a function of beam current is presented in Fig. 2. Figure 3 shows the correlated evolutions of the carrier concentrations at the $G B\left(n_{b}\right.$ and $\left.p_{b}\right)$ and at the edge of the SCR $\left(\Delta n_{0}\right)$. Three regimes successively occur as a function of excitation rate.

* Regime 1 corresponds to the so-called low injection regime. It is characterized by the condition $n_{b} \ll p_{b}$. This regime only appears in case (a) (low barrier) and for current below $\simeq 10^{-13} \mathrm{~A}$ (Figs.2a and $3 \mathrm{a}$ ). This gives a first important result : standard GBs in Si - which have barrier heights above $0.2 \mathrm{eV} / 5 /$ - cannot be studied in the low injection regime in usual EBIC experiments. In our example (b) $\left(\varphi_{b o}=0.28 \mathrm{eV}\right)$, the majority and minority carrier populations are already inversed at $\mathrm{I}_{\mathrm{b}}=10^{-13} \mathrm{~A}$ (Fig.3.b).

* Regime 2 is an intermediate regime, which corresponds to the experimental conditions most often used $\left(I_{b} \simeq 10^{-10} \mathrm{~A}\right)$.

The recombination velocity monotonously decreases with increasing injection. Fig.2 shows that this behaviour was already correctly described in our previous model (which indeed well fitted experimental results /1/) (dotted curves in Fig.2). The slight discrepancy at low currents in case (b) is due to the fact that the absolute limit of recombination velocity was arbitrarily put equal to the thermal velocity in $/ 1 /\left(\simeq 10^{7} \mathrm{~cm} \mathrm{~s}^{-1}\right)$ while it comes out of the calculation in the present work ( $v_{s}$ $\left.(1 \mathrm{im})=2.8 \times 10^{6} \mathrm{cms}^{-1}\right)$. Replacing the imposed value by the calculated one corrects the discrepancy.

* Regime 3 corresponds to the cases where the presence of carriers in the GB space charge cannot be schematically described, model /1/ does not apply anymore. This region is surprisingly characterized by a behaviour of the recombination velocity similar to that observed in the low injection case : the absence of dependence on excitation rate. This brings a second important result. The experimental observation of a constant recombination velocity over a narrow beam current range cannot be taken as a proof a low injection, one must investigate a range wide enough to include regime 2. Fig. 3 indicates that electron and hole concentrations are equal in regime 3 (and equal to the excess carrier concentration), which corroborates the quasi anihilation of the barrier (Fig.2). of course our description of the charge transport in the neutral region which considers the existence of majority and minority carriers is particularly weak in this case. However, considering ambipolar diffusion is not likely to change the behaviour observed.

Future work could be directed to a better treatment of this transport problem. However, carrying out experiments in the high injection range - which can easily be performed in any SEM - seems to be a priority.

\section{REFERENCES.}

/1/ MARFAING, Y. and MAURICE, J.-L., to be published in Springer Proc. Phys., 54 (1991).

/2/ EVERHART, T.E., and HOFF, P.M., J. Appl. Phys., 42 (1971) 5837.

/3/ SHOCKLEY, W., and READ Jr, W.T., Phys. Rev. 87 (1952) 835.

/4/ HALL, R.N., Phys. Rev. 87 (1952) 387.

/5/ GROVENOR, C.R.M. (Review), J. Phys. C 18 (1985) 4079. 